\title{
Numerical Study of the Effect of Thermosiphon on the Vertical Dispersion of Pollutants
}

\author{
Zeinebou Yahya $^{1}$ and Cheikh Mbow ${ }^{2}$ \\ 1. Department of Physics, Faculty of Sciences and Arts, Qassim University, Buraidah 51452, Kingdom of Saudi Arabia \\ 2. Department of Physics, Faculty of Science and Technology, Cheikh Anta Diop of Dakar University, Dakar 61641, Sengal
}

\begin{abstract}
The dispersion of pollutants in urban environments is a major problem. Because of this fact our study comes to propose prevention and security system against the dispersion of releases and fumes from industrial smokestacks. The system is modeled by a plume-thermosiphon interaction flow resulting from a vertical cylinder open at both ends with a generating source of the plume centered at the opening of the cylinder. The cylindrical wall heats under the effect of the thermal radiation emitted by the generator source, which causes a flow of thermosiphon around the flow of the thermal plume. In order to determine the radiosity of radiation leaving the wall we have been used the Simpson quadrature and the Gaussian elimination method with a dominant pivot. Numerical simulations have been executed for different positions of the thermosiphon compared to the plume source, and for a number of Rayleigh fixed $\mathrm{Ra}=10^{+7}$, and a radius of the hot source corresponds to $\mathrm{R}^{*}=0.46$. The results of the numerical model that we have been developed, help first identify the behavior of pollutants from the dynamic and thermal point of view and then explore the different locations of the thermosiphon compared to the source of the plume which causes a major influence on the dispersion of pollutants.
\end{abstract}

Key words: Interaction, numerical modelling, pollutants, thermosiphon.

\section{Introduction}

The thermal plume generated by the presence of a source of heat in an environment constrained by walls can simulate several cases in the industrial field. This is the case for example of hot air releases of the smokestacks of the fire in the stairwells or elevators and steam of thermal power plants, the dispersion of these pollutants in urban environments requires prevention and security systems. In this context, we propose to numerically model the spread of pollutants from industrial smokestacks, through a completely new configuration (Fig. 1). We have surrounded the thermal plume induced by a source of heat, by a cylindrical thermosiphon to determine the characteristics of the resulting flow. This system consists of a vertical cylinder open at both ends with a generating source of

Corresponding author: Zeinebou Yahya, Ph.D, assistant professor, research fields: numerical modeling (CFD), and heat transfer. the plume centered at the opening of the cylinder. The cylindrical wall heats under the effect of the thermal radiation emitted by the generator source, which causes a flow of thermosiphon around the flow of the thermal plume.

Several numerical and experimental studies have been conducted separately on the free thermal plume flows and unlimited [1,2], or the flows of thermosiphons [3-5] represented in our study by a cylinder heated by the effect of the previously mentioned radiation. However we note the crucial lack of numerical studies on interaction plume-thermosiphon which allows to better model our real case of the dispersion of the pollutants studied in this work. This problem is difficult to solve because writing input/output boundary conditions is a very delicate problem.

This study is inspired by the experimental study of Mahmoud et al. [6] which studied experimentally the effects of the thermosiphon on the dispersion of pollutants. 


\section{Theoretical Model}

The flow of interaction plume-thermosiphon studied in this work is outlined in Fig. 1.

Initially the system is in thermodynamic equilibrium at temperature $\mathrm{T}_{0}$. From time $\mathrm{T}_{0}$, the generator source is brought to a temperature and thus a natural convection takes birth that we propose to study. To do so we use the following simplifying assumptions:

- fluid Newtonian, viscous and transparent;

- laminar;

- unsteady flow;

- symmetric and two-dimensional flow to the axis of the cylinder;

- physical properties are constant except the density in the equation of motion;

- working pressure forces and the viscous dissipation are negligible.

The theoretical model is formulated from the equations of conservation of mass, the balance sheet of the movement amount and the heat equation, also by the initial and boundary conditions. Then we made this mathematical model dimensionless to show the characteristic quantities and to generalize the problem under study.

The dimensionless equations that govern the transfers in the system studied and their associated boundary conditions are given in Ref. [7].

\subsection{Radiosity in the Wall}

The radiative flux emitted by the generator source is divided into three parts, a part is recovered by the walls of the cylinder, and the other two parties are lost through the top and bottom of the cylinder openings.

The radiosity of radiation leaving the wall is given by

$$
J_{p}(R, z)=\varepsilon_{P} \sigma T_{P}^{4}+\left(1-\varepsilon_{P}\right) \sum_{i=1}^{L} \int_{S_{l}} J_{l} K\left(\vec{r}_{P}, \vec{r}_{l}\right) \cdot d S_{l}
$$

Where $\vec{r}_{1}$ are the positions of the elementary surfaces $d S_{p}, d S_{l}$ and $K\left(\vec{r}_{p} ; \vec{r}_{1}\right)$ the kernel defined by

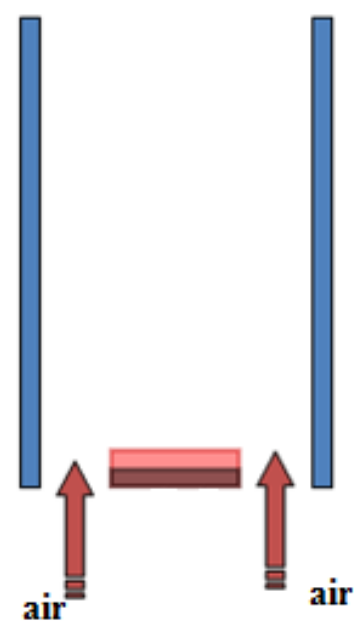

Fig. 1 Configuration studied.

$$
K\left(\vec{r}_{p} ; \vec{r}_{l}\right)=\frac{\left(\vec{n}_{p}, \vec{r}_{p, l}\right) \cdot\left(\vec{n}_{l} \cdot \vec{r}_{l, p}\right)}{\pi \cdot\left|\vec{r}_{p, l}\right|^{4}}
$$

With $\vec{n}_{p}$ and $\vec{n}_{l}$ the normal unit respectively of $d S_{p}$ and $d S_{l}$. In this relationship we asked $\vec{r}_{p, l}=-\vec{r}_{l, p}=\vec{r}_{l}-\vec{r}_{p}$. Considering the input and output as well as the source as black surfaces at temperature $\mathrm{T}_{\mathrm{a}}$ and $\mathrm{T}_{\mathrm{s}}$ it comes

$$
J_{p}=\varepsilon_{p} \sigma T_{p}^{4}+\left(1-\varepsilon_{p}\right)\left\{\begin{array}{l}
T_{a}^{4}\left[\int_{S_{e}} K\left(\vec{r}_{p}, \vec{r}_{e}\right) \cdot d S_{e}+\int_{S_{o}} K\left(\vec{r}_{p}, \vec{r}_{o}\right) \cdot d S_{o}\right]+ \\
T_{s}^{4} \int_{S_{l}} K\left(\vec{r}_{p}, \vec{r}_{s}\right) \cdot d S_{s}+\int_{S_{p}} J_{p}, K\left(\vec{r}_{p}, \vec{r}_{p^{\prime}}\right) \cdot d S_{p^{\prime}}
\end{array}\right\}
$$

The last term of this relationship translates the radiative exchange inter-walls. In the relations above the indices (e), (o) and (s) refer respectively to the input, output and the source. Indices (p) and ( $p^{\prime}$ ) are relative to the wall.

To simplify the writing we put our inhomogeneous integral equation known as Fredholm equation of the second order in the condensed form following

$$
J_{p}\left(\vec{r}_{p}\right)=J_{o p}\left(\vec{r}_{p}\right)+\lambda \int_{S^{\prime}} J_{p}\left(\vec{r}_{p^{\prime}}\right) \cdot K\left(\vec{r}_{p}, \vec{r}_{p^{\prime}}\right) \cdot d S^{\prime}
$$

With

$$
\lambda=-\left(1-\varepsilon_{p}\right)
$$

and

$$
J_{o p}\left(\vec{r}_{p}\right)=\varepsilon_{p} \sigma T_{p}^{4}+\left(1-\varepsilon_{p}\right)\left\{\begin{array}{l}
T_{a}^{4}\left[\int_{S_{e}} K\left(\vec{r}_{p}, \vec{r}_{e}\right) \cdot d S_{e}+\int_{S_{o}} K\left(\vec{r}_{p}, \vec{r}_{o}\right) \cdot d S_{o}\right]+ \\
T_{s}^{4} \int_{S_{l}} K\left(\vec{r}_{p}, \vec{r}_{s}\right) \cdot d S_{S}
\end{array}\right\}
$$


To solve this integral equation we approach the term $\int_{S^{\prime}} J_{p}\left(\vec{r}_{p^{\prime}}\right) \cdot K\left(\vec{r}_{p}, \vec{r}_{p^{\prime}}\right) \cdot d S^{\prime}$ using a quadrature Simpson and we get a system of equations that we are going to solve by a direct method (see the following paragraph).

\section{Numerical Model}

As transfers are more intense in the vicinity of the walls and at the input and output of the cylinder, there are discontinuities, so the field should be discretized using variable steps to minimize errors, the discretization of the balance sheets of the dimensionless equations and associated conditions is given in Ref. [7]. To solve the system of discretized equations, we use iterative methods that are based on the repeated application of a simple algorithm leading to the possible convergence after a finite number of repetition (iterations). We will use in this work iterative relaxation method line by line of Gauss-Seidel.

The calculation algorithm is structured as follows:

- Initiate speed, temperature and pressure fields;

- Calculate the coefficients of the equations of momentum and prediction rates: nicklist—speeds;

- Calculate the pressure step-by-step projection;

- Update the velocity field: calculate the corrected speed;

- Calculate the scope of temperatures.

In order to determine the radiosity of radiation leaving the wall where the radiative exchange of surface is taken into account, we put the radiosity in the following form

$$
J_{p}(R, z)-\int_{0}^{1} J_{p}\left(R, z^{\prime}\right) \cdot K\left(z, z^{\prime}\right) \cdot d z^{\prime}=f(z)
$$

Using the Simpson quadrature we have

$$
\int_{0}^{1} J_{p}\left(R, z^{\prime}\right) \cdot K\left(z, z^{\prime}\right) \cdot d z^{\prime} \approx \sum_{k=1}^{\left(n_{-} z-1\right) / 2} I_{2 k}(z)
$$

with

$$
I_{2 k}(z)=A_{2 k} F_{2 k-1}(z)+B_{2 k} F_{2 k}(z)+C_{2 k} F_{2 k+1}(z)
$$

The $F_{2 k}(Z)$ are given by: $F_{2 k}(Z)=J_{p, 2 k} \cdot K_{2 k}(Z)$ by posing $J_{p}(Z)=J_{i} ; K_{2 k}(Z)=K_{2 k, i} ; f(Z)=f_{i}$ we get the matrix system of $n_{-} \mathrm{z}$ equations in $\mathrm{n}_{-} \mathrm{z}$ unknowns

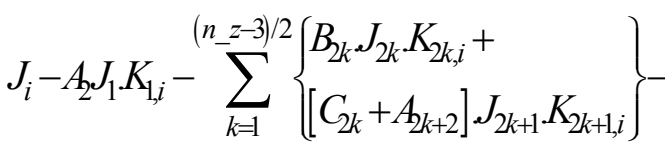

$$
\begin{aligned}
& B_{n_{-} z-1} J_{n_{-} z-1} \cdot K_{n_{-} z-1, i}-C_{n_{-} z-1} J_{n_{-} z} \cdot K_{n_{-} z, i}=f_{i}
\end{aligned}
$$

with $\mathrm{i}=1, \ldots \ldots \ldots, \mathrm{n}_{\mathrm{z}}$

This system is then solved by the Gaussian elimination method with a dominant pivot. Once the field radiosity determined we can calculate the local net radiative flux on the cylinder wall.

\section{Numerical Results and Discussion}

This section presents the results from the computer code that we have developed and which simulates the thermosiphon effect on the flow of thermal plume which represents pollutants.

\subsection{Dynamic and Thermal Fields in Transient State}

The curves of Figs. 2-5 show the variations of the longitudinal speed and temperature as a function of the radial coordinate for different times to $R a=$ $10^{7}$ and $R^{*}=0.46$ which correspond to a dimensionless radius of the source equal to 0.069 . During the first moments, the flow of boundary layer type near the wall does not affect the behavior of the fluid which is just at the top of the heat source. As time increases, the transfers are intensifying except in the vicinity of the area between the wall and the source where the temperature remains almost constant and therefore, the seat of a flow "return" that increases over time. The thickness of the hydrodynamic boundary layer developing interacts with the flow in the central area which undergoes increasingly the effect of the thermosyphon which results in a lateral displacement of the maxima of the longitudinal speed to the center of the enclosure. The analysis of the curves shows that the thermal steady settles more quickly than the hydrodynamic regime. 
In fact, the curves of Figs. 3 and 5 show that the fields of temperatures remain constant when $\mathrm{t} \geq 0.519$ while whose of delay $t=0.729$, you can consider that the longitudinal velocity profiles are self-similar (see Figs. 2 and 4).

\subsection{Effect of the Location of the Thermosiphon}

We study the influence of the location of the thermosiphon compared to the source of the plume (Fig. 6).

The curves in Fig. 7 show the variations of longitudinal velocity according to the coordinated radial when $\mathrm{h}$ (the vertical distance between the entrance of the thermosiphon and plume source) equal to 0.01

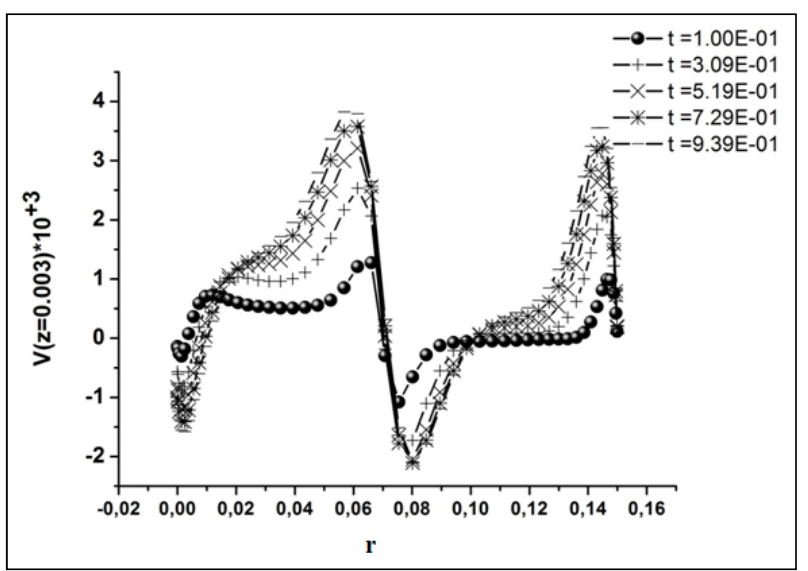

Fig. 2 Variation of the longitudinal speed as function of the dimensionless radial coordinate at $z=0.0029$ at different time.

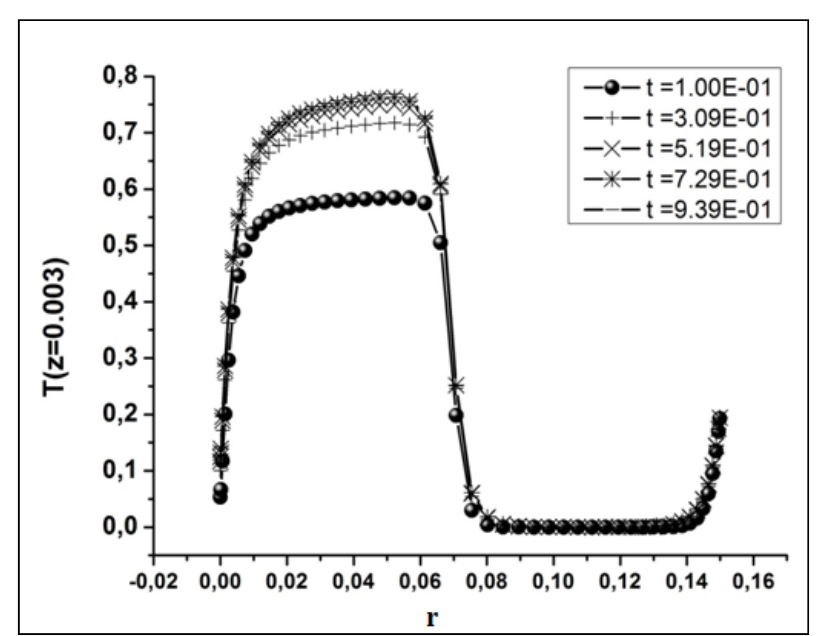

Fig. 3 Variation of the dimensionless temperature as function of the dimensionless radial coordinate at $\mathrm{z}=\mathbf{0 . 0 0 2 9}$ at different time.

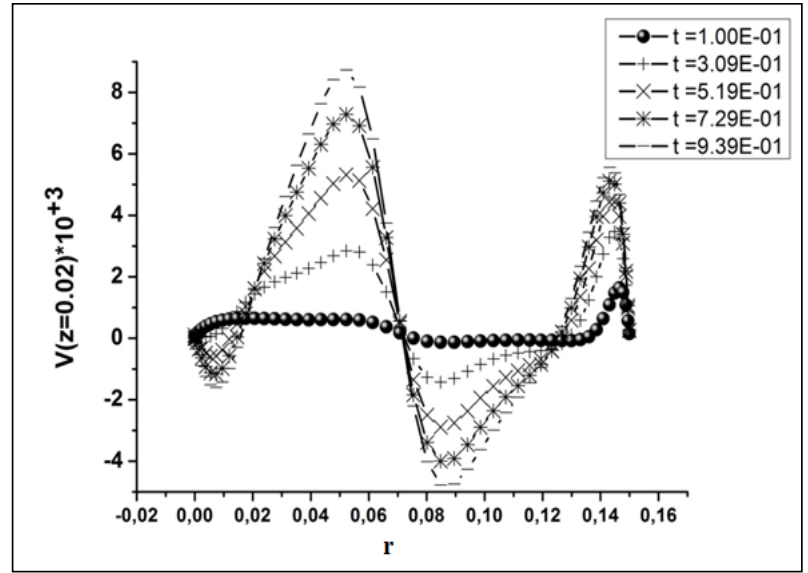

Fig. 4 Variations of the longitudinal speed as function of the dimensionless radial coordinate at $\mathrm{z}=\mathbf{0 . 0 2}$ at different moments.

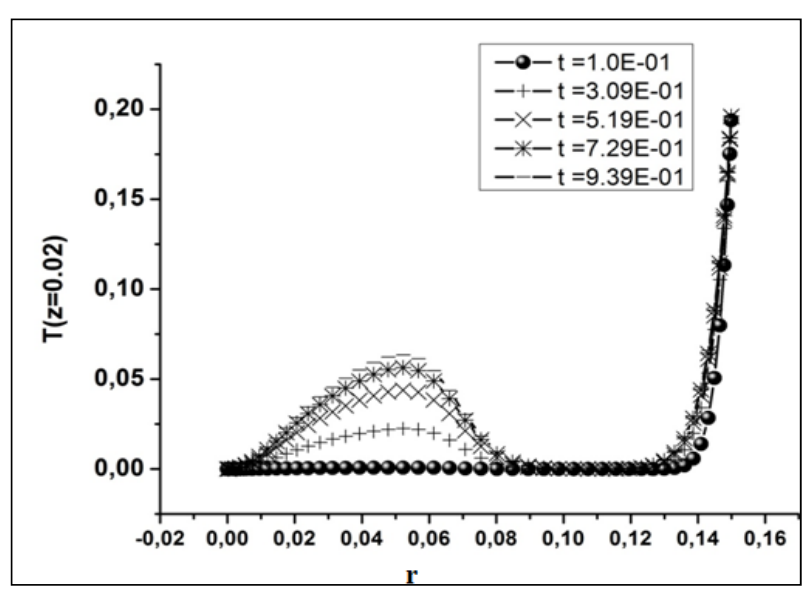

Fig. 5 Variations of the dimensionless temperature as function of the dimensionless radial coordinate at $\mathrm{z}=\mathbf{0 . 0 2}$ at different time.

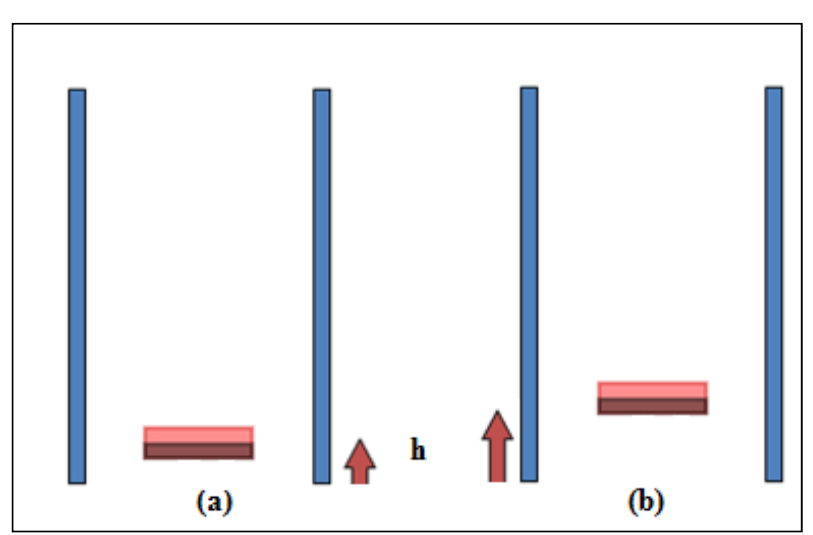

Fig. 6 Location of the thermosiphon compared to the source of the plume.

and 0.1 successively. And the curves of Fig. 8 show the variations of temperature according to the coordinated radial when h equal to 0.01 and 0.1 successively. 

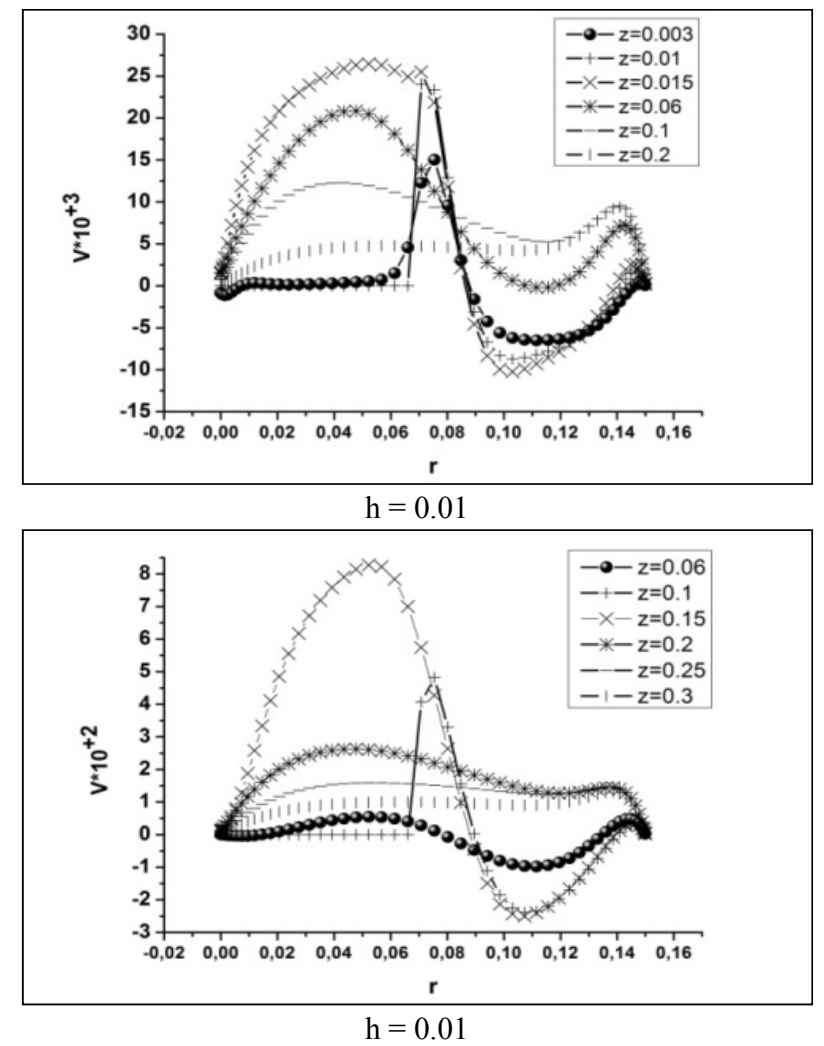

Fig. 7 Variations of the longitudinal velocity versus of the radial coordinate at $h=0.01$ and $h=0.1$.
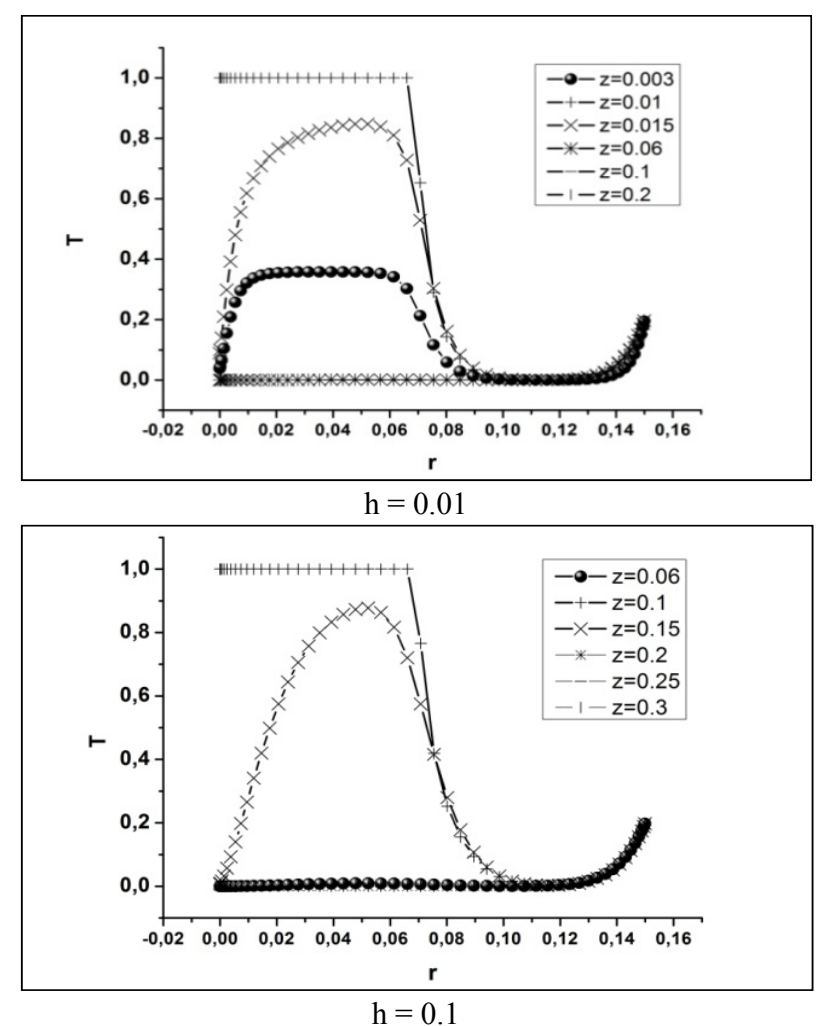

Fig. 8 Variations of the temperature versus of the radial coordinate at $h=0.01$ and $h=0.1$.
The curves in Fig. 8 show that the effects of the thermosiphon increase, gradually as the hot source is penetrated into the cylinder (industrial chimney), and that the maximum move toward the wall. Above the source increasing the vertical distance (h) between the inlet of the thermosiphon and the hot source causes an increase in speed and a rapid homogenization of the fluid.

\section{Conclusions}

In this work we have modeled the real problem of propagation of pollutants using the thermal plume limited by cylindrical walls, this problem has been represented by an equation system of Navier-Stokes equations and equation of the heat, and by initial conditions and limits, despite the difficulty of writing policies to limits input/output for the problems related to the thermosiphon.

Numerical simulations have been executed for different positions of the thermosiphon compared to the plume source, and for a number of Rayleigh fixed $\mathrm{Ra}=10^{+7}$, and a radius of the hot source corresponds to $\mathrm{R}^{*}=0.46$.

The study has determined the mechanisms of interaction of the two flows of thermal plume and thermosiphon, it also showed that the position of the thermosiphon compared to the plume source has a major influence on the dispersion of pollutants.

\section{References}

[1] Guillou, B. 1984. "Etude Numérique et expérimentale de la structure turbulente d'un panache pur à symétrie axiale." Ph.D. thesis, Poitiers University.

[2] Agator, J. M., and Doan-Kim-Son. 1983. "Analyse spectral du champ thermique d'un panache turbulent à symétrie axiale." C. R. Acad. Sc. Paris 296 (II): 1119-22.

[3] Kettleborough, C. 1972. "Transient Laminar Free Convection between Heated Vertical Plates including Entrance Effects." International Journal of Heat and Mass Transfer 15: 883-96.

[4] Wirtz, R., and Stutzman, R. 1982. "Experiments on Free Convection between Vertical Plates with Symmetric Heating." Journal of Heat Transfer, 104.

[5] Ramanathan, S., and Kumar, R. 1991. "Correlations for 
Natural Convection between Heated Vertical Plates." Journal of Heat Transfer (Transcations of the ASME (American Society of Mechanical Engineers), SeriesC, United States 113.

[6] Mahmoud, A. O. M., Zinoubi, J., Ben Maad, R., and Belghith, A. 2006. "Improvement of the Vertical Dispersion of Pollutants Resulting from Chimneys by Thermosiphon Effect." American Journal of Environmental Sciences 2: 66-73.
[7] Yahya, Z., Mbow, Ch., Mahmoud, A. O. M., and Sow, M. L. 2013. "Modeling of a Natural Convection Flow Plume -Thermosiphon Interaction." Int. Rev. of Mechanical Enginneering 7: 140-6.

[8] Zinoubi, J. 2003. "Etude de l'interaction d'un écoulement de thermosiphon avec un panache thermique à symétrie axiale : influences des paramètres de forme." Thèse de doctorat, Université de Tunis II, Faculté des sciences de Tunis. 